a variant EMT phenotype induced by the tumor-specific antigen TM4SF5.

Address correspondence to: Ruth J. Muschel, Radiation Oncology and Biology, The Radiobiology Research Institute, Churchill Hospital, University of Oxford, Oxford OX3 7LJ, United Kingdom. Phone: 44-01865-857-427; Fax: 44-0-1865-857-533; E-mail: ruth.muschel@rob.ox.ac.uk.

1. Gress, T.M., et al. 1997. Identification of genes with specific expression in pancreatic cancer by cDNA representational difference analysis. Genes Chromosomes Cancer. 19:97-103.

2. Muller-Pillasch, F., et al. 1998. Identification of a new tumour-associated antigen TM4SF5 and its expression in human cancer. Gene. 208:25-30.

3. Wright, M.D., Ni, J., and Rudy, G.B. 2000. The L6 membrane proteins - a new four-transmembrane superfamily. Protein Sci. 9:1594-1600.

4. Chang, Y.W., et al. 2005. CD13 (aminopeptidase N) can associate with tumor-associated antigen L6 and enhance the motility of human lung cancer cells. Int. J. Cancer. 116:243-252.

5. Lazo, P. 2007. Functional implications of tetraspanin proteins in cancer biology. Cancer Sci. 98:1666-1677.

6. Hay, E.D. 1995. An overview of epithelio-mesenchymal transformations. Acta Anat. 154:8-20.

7. Kiemer, A.K., Takeuchi, K., and Quinlan, M.P. 2001. Identification of genes involved in epithelialmesenchymal transition and tumor progression.
Oncogene. 20:6679-6688

8. Janda, E., et al. 2002. Ras and TGF[beta] cooperatively regulate epithelial cell plasticity and metastasis: dissection of Ras signaling pathways. J. Cell Biol. 156:299-313

9. Vincent-Salomon, A., and Thiery, J.P. 2003. Host microenvironment in breast cancer development: epithelial-mesenchymal transition in breast cancer development. Breast Cancer Res. 5:101-106.

10. Xue, C., Plieth, D., Venkov, C., Xu, C., and Neilson, E.G. 2003. The gatekeeper effect of epithelialmesenchymal transition regulates the frequency of breast cancer metastases. Cancer Res. 63:3386-3394.

11. Hemler, M.E. 2005. Tetraspanin functions and associated microdomains. Nat. Rev. Mol. Cell Biol. 6:801-811.

12. Lammerding, J., Kazarov, A.R., Huang, H., Lee, R.T., and Hemler, M.E. 2003. Tetraspanin CD151 regulates alpha6beta1 integrin adhesion strengthening. Proc. Natl. Acad. Sci. U. S. A. 100:7616-7621.

13. Garcia-Lopez, M.A., et al. 2005. Role of tetraspanins CD9 and CD151 in primary melanocyte motility. J. Invest. Dermatol. 125:1001-1009.

14. Testa, J.E., Brooks, P.C., Lin, J.M., and Quigley, J.P. 1999. Eukaryotic expression cloning with an antimetastatic monoclonal antibody identifies a tetraspanin (PETA-3/CD151) as an effector of human tumor cell migration and metastasis. Cancer Res. 59:3812-3820.

15. Lee, S.-A., et al. 2008. Tetraspanin TM4SF5 mediates loss of contact inhibition through epithelialmesenchymal transition in human hepatocarcinoma. J. Clin. Invest. 118:1354-1366.

16. Lee, S.Y., et al. 2006. Focal adhesion and actin organization by a cross-talk of TM4SF5 with integrin alpha2 are regulated by serum treatment. Exp. Cell Res. 312:2983-2999.
17. Gal, A., et al. 2008. Sustained TGFbeta exposure suppresses Smad and non-Smad signalling in mammary epithelial cells, leading to EMT and inhibition of growth arrest and apoptosis. Oncogene. 27:1218-1230.

18. Gotzmann, J., et al. 2004. Molecular aspects of epithelial cell plasticity: implications for local tumor invasion and metastasis. Mutat. Res. 566:9-20.

19. Kaimori, A., et al. 2007. Transforming growth factor-beta 1 induces an epithelial-to-mesenchymal transition state in mouse hepatocytes in vitro. J. Biol. Chem. 282:22089-22101.

20. Gotzmann, J., et al. 2002. Hepatocytes convert to a fibroblastoid phenotype through the cooperation of TGF-beta1 and Ha-Ras: steps towards invasiveness. J. Cell Sci. 115:1189-1202.

21. Huber, M.A., Kraut, N., and Beug, H. 2005. Molecular requirements for epithelial-mesenchymal transition during tumor progression. Curr. Opin. Cell Biol. 17:548-558.

22. Moustakas, A., and Heldin, C.H. 2007. Signaling networks guiding epithelial-mesenchymal transitions during embryogenesis and cancer progression. Cancer Sci. 98:1512-1520.

23. Bakin, A.V., Tomlinson, A.K., Bhowmick, N.A., Moses, H.L., and Arteaga, C.L. 2000. Phosphatidylinositol 3-kinase function is required for transforming growth factor beta-mediated epithelial to mesenchymal transition and cell migration. J. Biol. Chem. 275:36803-36810.

24.Yang, Y., et al. 2006. Regulation of transforming growth factor- $\beta 1$-induced apoptosis and epithelial-to-mesenchymal transition by protein kinase A and signal transducers and activators of transcription 3. Cancer Res. 66:8617-8624.

\title{
HOXB4 and retroviral vectors: adding fuel to the fire
}

\author{
Andre Larochelle and Cynthia E. Dunbar
}

Molecular Hematopoiesis Section, Hematology Branch, National Heart, Lung, and Blood Institute, National Institutes of Health, Bethesda, Maryland, USA.

\begin{abstract}
The transcription factor homeobox B4 (HOXB4) is a promising agent capable of providing a growth advantage to genetically modified hematopoietic stem and progenitor cells (HSPCs). In this issue of the JCI, Zhang and colleagues overexpressed HOXB4 in HSPCs from large animals using retroviral vectors (see the related article beginning on page 1502). Two years after transplantation, most animals developed leukemia, a consequence of combined HOXB4 and deregulated protooncogene expression. These results highlight the risks of combining integrating vectors and growth-promoting genes for clinical applications.
\end{abstract}

\section{Hematopoietic stem cells and gene therapy}

Hematopoietic stem and progenitor cells (HSPCs) are ideal targets for permanent

Nonstandard abbreviations used: HOXB4, homeobox B4; HSPC, hematopoietic stem and progenitor cell; IL2RG, IL-2 receptor $\gamma$; LMO2, LIM domain only 2; SCID-X, X-linked SCID.

Conflict of interest: The authors have declared that no conflict of interest exists.

Citation for this article: J. Clin. Invest. 118:1350-1353 (2008). doi:10.1172/JCI35326. genetic correction of defects in any lineage of hematopoietic cells. Most clinical applications have used gene transfer vectors based on integrating retroviruses, but the relative inefficiency of these vectors has limited the considerable potential of gene transfer into HSPCs. Genetically modified cells represent only a small fraction $(1 \%-10 \%)$ of the hematopoietic cells after transplantation. Consequently, competition from unmodified infused and endogenous HSPCs may dilute any therapeutic effect of the transduced cells. This implies that this small fraction of genetically modified hematopoietic cells will correct diseases requiring more than $1 \%-10 \%$ of corrected cells only if they have a marked selective growth advantage in vivo.

\section{Homeobox B4 promotes a selective growth advantage of transduced HSPCs}

In most diseases considered to be suitable targets for gene therapy, corrected cells do not themselves have an inherent growth advantage, providing an impetus to arm retroviral vectors with genes capable of conferring a selective growth advantage to transduced HSPCs and their progeny in vivo. Ectopic expression of homeobox B4 (HOXB4), a transcription factor containing a highly conserved DNA-binding motif known as the homeodomain, has been found to enhance HSPC self-renewal in vitro and in vivo and has been suggested as an 
A

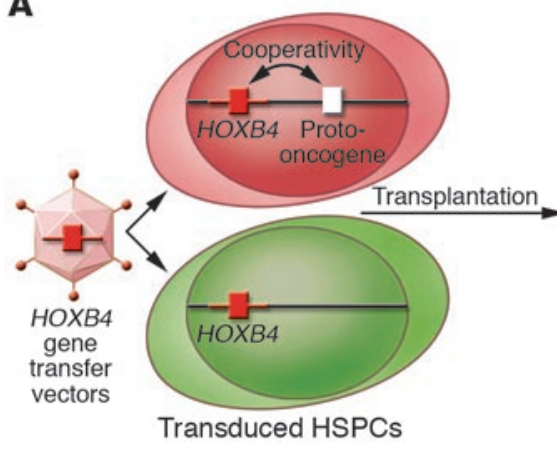

B

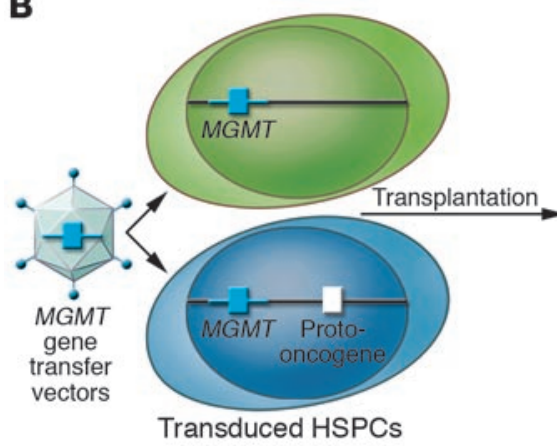

Figure 1

Development of leukemia after transplantation of HSPCs transduced with retroviral vectors. (A) In rare HSPCs, HOXB4-containing vectors integrate near protooncogenes and the influence of the vector's strong promoter deregulates their expression, favoring the engraftment and proliferation of these clones over those without activated protooncogenes. The additional expression of HOXB4 may give these clones further proliferative advantage compared with those without HOXB4. These expanded clones subsequently enter a dormant phase (the latency period), when new mutations accumulate in their genome. This increased tendency to accumulate DNA damage may be related to the deregulated expression of protooncogenes within these clones. Multiple-hit models of tumorigenesis predict that the higher number of cells initially expanded under the influence of HOXB4 increases the likelihood that one or several of these cells will acquire an additional mutation (M), leading to uncontrolled clonal expansion and overt leukemia. (B) In the absence of HOXB4 or similar growth-promoting genes in the gene transfer vector (for example, with vectors containing the drug selection gene methylguanine methyltransferase [MGMT], as used in the study in this issue by Zhang et al.; ref. 19), integration near protooncogenes is possible, leading to their deregulation due the influence of the vector's strong promoter. However, in the absence of cooperativity from HOXB4 (or other growth-promoting genes) in the gene-transfer vector, initial proliferation of these clones is less pronounced and, therefore, the risk that one or several of these cells will acquire an additional mutation, leading to uncontrolled clonal expansion and overt leukemia, is lower.

approach not only to enhance gene therapy efficacy, but also in other clinical settings where the numbers of HSPCs are limited, such as cord blood transplantation.

The impact of HOXB4 expression on HSPC self-renewal was discovered by Sauvageau et al., who demonstrated that transfection of normal mouse bone marrow cells with a retroviral vector overexpressing HOXB4 resulted in a dramatic increase in engraftment ability and HSPC number compared with marrow transfected with a control vector (1). Importantly, the recipient mice did not develop hematopoietic abnormalities or malignancies, suggesting HOXB4-expressing HSPCs were still subject to normal homeostatic controls $(2,3)$. Since these initial studies, it has additionally been reported that HOXB 4 can expand primitive human progenitors in culture as well as those human cells able to engraft immunodeficient mice $(4,5)$.

\section{HOXB4 and leukemic transformation: the time has come}

Despite these encouraging findings, investigators have worried that inclusion of HOXB4 in gene transfer vectors could carry significant risks, particularly in the context of evolving concerns regarding vector-related insertional mutagenesis $(6,7)$. The strong enhancers contained in retroviral genomes can activate adjacent cellular genes following integration, but the risk posed by one or a few integrated vectors per cell was estimated to be very low. However, these assumptions were shattered in 2002, when French researchers announced that a child who had shown unequivocal clinical benefit in the HSPC gene therapy trial for X-linked SCID (SCID-X) $(8,9)$ developed a vector-related $\mathrm{T}$ cell acute leukemia three years after infusion of retrovirally modified CD $34^{+}$cells (10). Since then, three other children in the French trial and, more recently, one patient in a similar SCID-X study conducted by British researchers have developed leukemias. In at least three cases, the tumor cells had a clonal vector insertion activating the LIM domain only 2 (LMO2) transcription factor gene (11). It was hypothesized that cooperation between LMO2 and the growth-promoting effect of the corrective IL-2 receptor $\gamma$ (IL2RG) transgene resulted in leukemic transformation, perhaps limiting the problem to SCID-X (12). However, 2 patients with chronic granulomatous disease treated in an HSPC gene therapy protocol have developed abnormal hematopoiesis and clonal expansion of vector-containing cells due to activation of protooncogenes (e.g., MDSEVI1), despite the lack of growth-promoting activity of the corrective transgene (13). Our group also reported the development of vector-associated myeloid sarcoma in a rhesus macaque five years following transplantation of HSPCs transduced with a vector containing only a marker gene and a drug-resistance gene (14).

It has been demonstrated that high-level HOXB4 expression, achieved with retroviral (5) or adenoviral (15) vectors in human $\mathrm{CD} 34^{+}$cells, perturbed the myeloid differentiation program both in vitro $(5,15)$ an in vivo (5) without frank leukemia. Similarly, disturbed myeloid differentiation was observed in a multipotent hematopoietic cell line in vitro (16). Ectopic HOXB4 expression also proved to be key in the production of primitive mouse hematopoietic cells from embryonic stem cells then able to engraft irradiated recipients; however, these mice had abnormal hematopoiesis with enforced myeloid and suppressed lymphoid development (17).

Despite these warning signs and continued concern regarding potential cooperativ- 
ity between growth-altering transgenes and vector insertion sites, investigators continued to pursue the potential of the HOXB4 approach. In a study published in 2006, Kiem and his research group at the Fred Hutchinson Cancer Research Center reported preliminary results in their powerful non-human primate competitive repopulation model (18). They transduced monkey $\mathrm{CD} 4^{+}$cells with either a HOXB4-expressing vector or a control vector expressing only a marker gene and analyzed the competitive repopulating ability of these cells in vivo. As hoped, there was a very significant advantage for the HOXB4-transduced cells early following engraftment of the monkeys, but in contrast to mouse studies, a much less significant advantage for the HOXB4-transduced cells was observed long-term after transplantation (18).

In their study in this issue of the JCI, the same group now reports the first instances of leukemia linked to HOXB4 expression, both in the original group of monkeys now followed longer term and in dogs that received cells transduced with a HOXB4expressing vector (19). Some 2 years after transplantation, 3 of 4 animals ( 2 of 2 dogs and 1 of 2 non-human primates) developed acute myeloid leukemias. Several lines of evidence strongly implicated HOXB4 in leukemogenesis. High-level HOXB4 expression was confirmed in the leukemic cells. Also, 3 of 4 animals followed long-term in this study developed leukemia, compared with none of more than 40 dogs and monkeys transplanted with cells transduced with similar vectors not expressing HOXB4 by the same research group and only 1 of 80 macaques followed long-term by our group (14). The most compelling evidence for the pivotal role of HOXB4 was provided by deriving a cell line from the leukemic cells of one animal and using short hairpin RNAs to knockdown HOXB4 expression in these cells. Profound growth inhibition and rapid cell death occurred with downregulation of the HOXB4 transgene.

Was HOXB4 expression sufficient as a single event to cause leukemia? To gain further insights, the authors localized vector integration sites in the genome (19). Interestingly, insertion sites in all tumors were near or within several protooncogenes, including c-myb and PRDM16 (already implicated in the chronic granulomatous disease trial described above), leading to aberrant expression of these genes. This suggests cooperativity between $\mathrm{HOXB} 4$ and the nearby activated protooncogenes, similar to the proposed cooperativity between $\mathrm{LMO} 2$ vector activation and the IL2RG transgene in the SCID-X trials. Overall, HOXB4 can be considered as a catalyst for leukemia development following retroviral gene transfer (Figure 1).

This study underscores the importance of large animal models in further development of gene and other novel HSPCdirected therapies. Perhaps no leukemias linked to HOXB4 overexpression developed in mice because too few gene-modified HSPCs could be given to these small animals, much fewer than the number of transduced HSPCs transplanted in clinical trials or larger animal models. Or perhaps a long latency period, longer than the lifespan of a mouse, was required before evidence of leukemic transformation could be seen. HOXB4 or similar growth-promoting genes are not a sine qua non for the development of malignancies following HSPC gene transfer, but they appear to significantly increase the probability of such an event.

\section{HOXB4 in HSPC gene therapy: time to abandon?}

The message of the current study by Zhang and colleagues (19) is clear as a bell: overexpression of HOXB4 using integrating retroviral vectors is much too risky to be contemplated in any clinical settings. This is not to say that this study rules out alternative approaches to harnessing the power of HOXB4 on HSPCs. For instance, a soluble recombinant HOXB4 protein induced rapid ex vivo expansion of transduced HSPCs, thereby avoiding the use of integrating retroviral vectors while benefiting from the self-renewal capacity of HOXB4 $(20,21)$. However, the short half-life of the recombinant protein has proven a practical hurdle, and it remains unclear whether HOXB4 can expand human and primate long-term hematopoietic stem cells. Alternatively, HOXB4 may be transiently delivered to cells using nonintegrating retroviral vectors. "Suicide genes" such as herpes thymidine kinase could be included in vectors to provide the opportunity for delivering an exogenous "mission abort" signal via administration of ganciclovir if evidence for unchecked clonal growth appeared following gene therapy. However, killing efficiency is unlikely to be complete and, therefore, many problematic clones and subclones could persist after ganciclovir treatment.

Events in the recent history of gene therapy have led many to acknowledge that the field may have been, on several occasions, too eager to rush into the clinic. However, the wisdom gained from these setbacks is now becoming apparent in studies such as that of Zhang and colleagues in this issue of the JCI (19). Scientists and clinicians hoping to use their protocols to treat humans understand the importance of examining vectors' behavior in a variety of comparative assays, including large animal studies, before entering the clinic. The use of well-characterized and well-tested preclinical models such as those of Zhang and colleagues is likely to minimize the risk of an adverse event that would throw the field into another crisis.

Address correspondence to: Cynthia E. Dunbar, National Heart, Lung, and Blood Institute, Hematology Branch, Building 10CRC, Room 4-5132, 10 Center Drive, Bethesda, Maryland 20892, USA. Phone: (301) 496-1434; Fax: (301) 496-8396; E-mail: dunbarc@nhlbi.nih.gov.

1. Sauvageau, G., et al. 1995. Overexpression of HOXB4 in hematopoietic cells causes the selective expansion of more primitive populations in vitro and in vivo. Genes Dev. 9:1753-1765.

2. Perkins, A.C., and Cory, S. 1993. Conditional immortalization of mouse myelomonocytic, megakaryocytic and mast cell progenitors by the Hox-2.4 homeobox gene. EMBO J. 12:3835-3846.

3. Thorsteinsdottir, U., et al. 1997. Overexpression of HOXA10 in murine hematopoietic cells perturbs both myeloid and lymphoid differentiation and leads to acute myeloid leukemia. Mol. Cell. Biol. 17:495-505.

4. Buske, C., et al. 2002. Deregulated expression of HOXB4 enhances the primitive growth activity of human hematopoietic cells. Blood. 100:862-868.

5. Schiedlmeier, B., et al. 2003. High-level ectopic HOXB4 expression confers a profound in vivo competitive growth advantage on human cord blood CD34+ cells, but impairs lymphomyeloid differentiation. Blood. 101:1759-1768.

6 . Baum, C., et al. 2004. Chance or necessity? Insertional mutagenesis in gene therapy and its consequences. Mol. Ther. 9:5-13.

7. Baum, C., et al. 2003. Side effects of retroviral gene transfer into hematopoietic stem cells. Blood. 101:2099-2114.

8. Cavazzana-Calvo, M., et al. 2000. Gene therapy of human severe combined immunodeficiency (SCID)-X1 disease. Science. 288:669-672.

9. Hacein-Bey-Abina, S., et al. 2002. Sustained correction of X-linked severe combined immunodeficiency by ex vivo gene therapy. N. Engl. J. Med. 346:1185-1193.

10. Hacein-Bey-Abina, S., et al. 2003. A serious adverse event after successful gene therapy for X-linked severe combined immunodeficiency. N. Engl. J. Med. 348:255-256.

11. Hacein-Bey-Abina, S., et al. 2003. LMO2-associated clonal $\mathrm{T}$ cell proliferation in two patients after gene therapy for SCID-X1. Science. 302:415-419.

12. Dave, U.P., Jenkins, N.A., and Copeland, N.G. 2004. Gene therapy insertional mutagenesis insights. Science. 303:333.

13. Ott, M.G., et al. 2006. Correction of X-linked chronic granulomatous disease by gene therapy, augmented by insertional activation of MDS1EVI1, PRDM16 or SETBP1. Nat. Med. 12:401-409. 
14. Seggewiss, R., et al. 2006. Acute myeloid leukemia is associated with retroviral gene transfer to hematopoietic progenitor cells in a rhesus macaque. Blood. 107:3865-3867.

15. Brun, A.C., Fan, X., Bjornsson, J.M., Humphries, R.K., and Karlsson, S. 2003. Enforced adenoviral vector-mediated expression of HOXB4 in human umbilical cord blood CD34+ cells promotes myeloid differentiation but not proliferation. Mol. Ther. 8:618-628.
16. Milsom, M.D., et al. 2005. Overexpression of HOXB4 confers a myelo-erythroid differentiation delay in vitro. Lenkemia. 19:148-153.

17. Pilat, S., et al. 2005. HOXB4 enforces equivalent fates of ES-cell-derived and adult hematopoietic cells. Proc. Natl. Acad. Sci. U. S. A. 102:12101-12106.

18. Zhang, X.-B., et al. 2006. Differential effects of HOXB4 on nonhuman primate short- and longterm repopulating cells. PLoS. Med. 3:e173.

19. Zhang, X.-B., et al. 2008. High incidence of leuke- mia in large animals after stem cell gene therapy with a HOXB4-expressing retroviral vector. J. Clin. Invest. 118:1502-1510.

20. Amsellem, S., et al. 2003. Ex vivo expansion of human hematopoietic stem cells by direct delivery of the HOXB4 homeoprotein. Nat. Med. 9:1423-1427.

21. Krosl, J., et al. 2003. In vitro expansion of hematopoietic stem cells by recombinant TAT-HOXB4 protein. Nat. Med. 9:1428-1432. 